\title{
Transport in the quantum world
}

\author{
Tadhg Morgan
}

Department of Physics, UCC

\section{Introduction}

The relentless progression of technology is something we are all familiar with. Computers have gone from filling entire rooms to only taking up some desk space while at the same time becoming incredibly fast. Music was once stored on vinyl records but we can now store hundreds of albums on portable MP3 players. This progression is described by Moore's law which says that technology is getting twice as small and twice as fast every eighteen months. However, this progression can only continue unhindered for so long until it hits a fundamental wall. The problem is that the miniaturization of technology is moving it out of the classical, everyday world and into the quantum world, and devices will soon reach the size of single or few atoms.

Whilst moving into the quantum world presents a number of challenges, the benefits far out weigh them. Quantum computers, computers which utilize quantum mechanics, represent a paradigm shift in computing. These are extremely fast machines that can tackle problems beyond the reach of current computers. However, there are many fundamental problems that need to be solved before these machines can be realized. We need to build a new quantum toolbox before we can begin engineering these machines. One of the most basic and essential tools we need for this tool box is transport, the ability to change an object's position in space. To develop a tool for transport in the quantum world we first need to understand two of the fundamental differences between the classical world and the quantum world.

\section{Quantum vs Classical}

We are all familiar with the behaviour of objects in the classical world but what changes in the quantum world? The key difference in the quantum world is that an object can no longer be though of as a just a particle, but as wave at the same time, and vise versa. This is what we call wave-particle duality. For example, light, which we usually think of as a wave, can be viewed as consisting of particles of light called photons. Similarly, atoms, usually thought of as particles, can produce effects we usually associate with waves. This has a huge impact on the transport of atoms because in the same way that water waves in the oceans interfere with each other, the atoms will also interfere with each other. 
Essentially what this means is that if you try and transport a quantum object such as an atom from $A$ to $B$, interference during transport will cause the object to spread out between $\mathrm{A}$ and $\mathrm{B}$.

The other major difference between the classical world and the quantum world is the idea of position. Instead of being at a well defined position as in the classical world, a quantum object has a certain probability of being at a position. Because of this transport from A to $B$ is the same as initially having a high probability of being at $A$ and then increasing its probability of being at $B$. But because of the wave-like interference spreading out the object's position, there is a probability of finding the object at $\mathrm{A}$, or $\mathrm{B}$, or any point in between.

This exact problem occurs when trying to transport atoms. This is a huge problem for developing quantum technology because, as you can imagine, transport is essential for building quantum devices. For most quantum devices our atoms are stored in traps or trapping potentials. These traps operate like valleys, it is easy to sit at the bottom, but a lot of energy is required to climb out. We can transport atoms between two traps by a process known as tunnelling, which occurs when two traps are moved closer together. Ideally, when the traps are moved apart again, the atom would have tunnelled from the initial trap to the destination trap, but the interference effects will prevent this from happening. All of this leads us to conclude that we cannot apply the classical approach to transport to quantum systems. We have to come up with new and different transport methods for the quantum world.

\section{My Research}

My research aims to develop a method of controlled quantum transport which will allow us to transport a quantum object without it spreading out among the different stages of transport. To do this we introduce an intermediate trap between the initial position and our destination. At first glance this would seem to make the problem harder. If we attempt to transport our quantum object in an intuitive way, bringing the initial trap close to the intermediate and then bringing the intermediate close to the destination, our quantum particle spreads out among all three traps. The benefit of having this intermediate trap, however, is that it enables us to perform counter-intuitive transport. We achieve this by bringing the destination close to the intermediate trap first without moving the initial trap. After this we bring the initial trap close to the intermediate. This method doesn't work in the classical world, you cannot get to work by walking from your car into your office, and then walking from your house into the car. In the quantum world, however, this works extremely well. Our numerical simulations have shown that by using this process, the end result is that the atom is in the destination trap and has no probability of being found in either of the other two traps. The graph below shows the probability the atom has for 


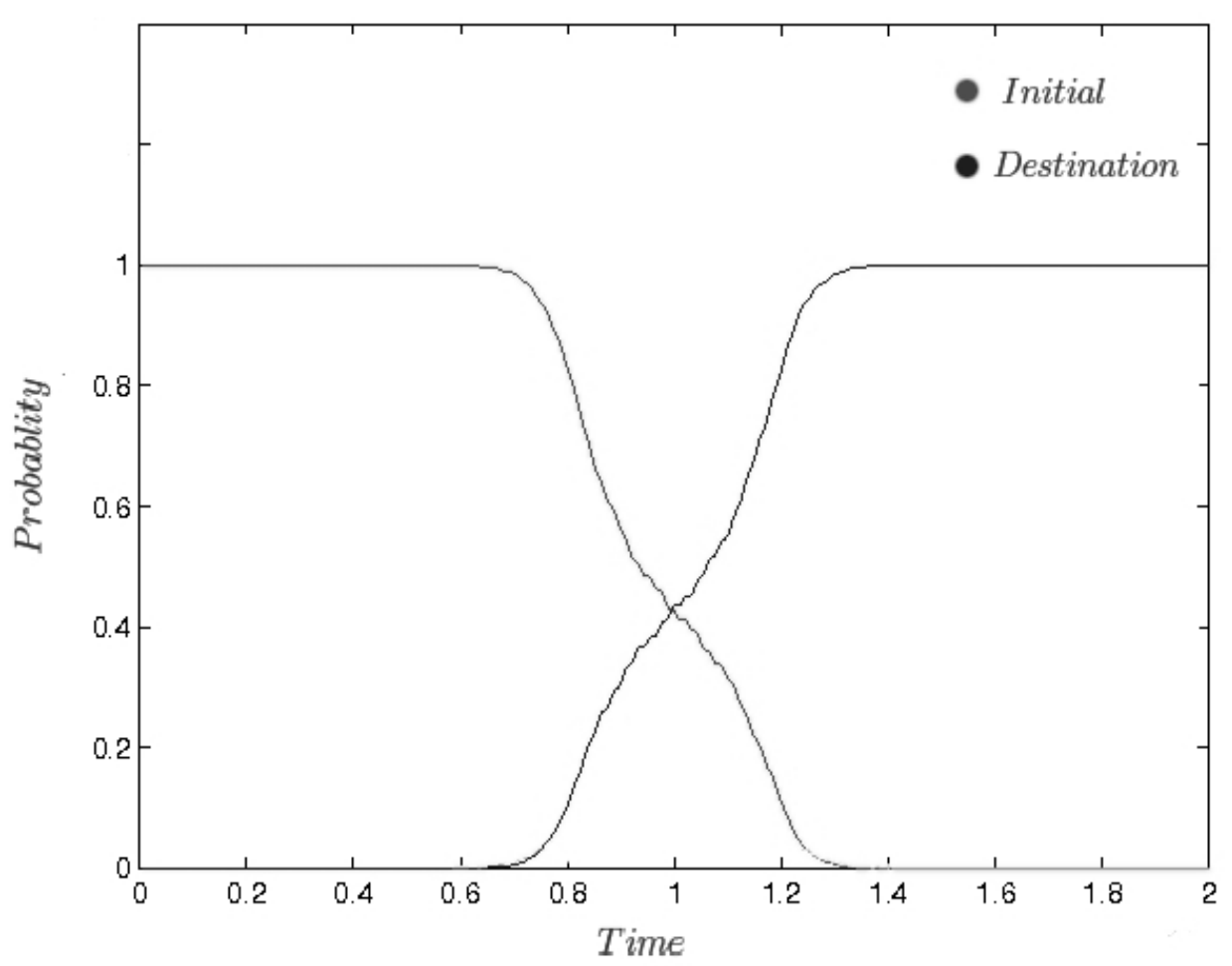

Figure 1: Probability the atom has for being in the initial trap (red line) and the destination trap (blue line) with time.

being in the initial trap (red line) and the destination trap (blue line) with time. From the graph you can also notice the at no time does the atom have any probability of being in the intermediate trap. We have also extended this beyond the mathematical description to radio frequency traps that are easily achievable in the lab.

Although we have the first tool for our tool box many more tools need to be added. A natural continuation of this research is to investigate methods of transporting clouds of atoms. The problem that needs to be solved is that the atoms in the cloud interact with each other. Like the interference effect that I have outlined in this article, interaction between the atoms disrupts the transport process. Another exciting idea that I would love to work on is investigating if the quantum transport method described above can be used with pairs of atoms to make logic gates, the building blocks of quantum computers.

This research is part of a large worldwide effort to develop tools for quantum engineering. We work in collaboration with The University of Nottingham on this counter-intuitive transport and also with ICHEC (Irish Centre for High End Computing) to run our simulations of these systems. As well as designing other tools, we need to investigate the counter-intuitive transport process in many other physical systems. This huge international effort into quantum engineering is due to the potential of quantum technology. It represents the next technology leap which will bring amazing new devices that will change 
our lives. Ireland has a long history of being a centre for new technology and innovative research. We aim to continue this tradition into the future with quantum technology.

Tadhg Morgan is a student in the Physics Department under the supervision of Dr. Thomas Busch. The author would like to acknowledge funding from Science Foundation Ireland. 